\title{
Alterações metabólicas, in vivo, induzidas por oclusão carotídea bilateral, no sangue e tecido ocular de ratos Wistar ${ }^{1}$
}

\author{
Eliane Silva de Oliveira ${ }^{2}$ \\ Sérgio Botelho Guimarães ${ }^{3}$ \\ Daniel Duarte Gadelha ${ }^{4}$ \\ Anselmo Martins de Araújo Filho ${ }^{4}$ \\ Paulo Roberto Leitão de Vasconcelos ${ }^{5}$
}

\begin{abstract}
Oliveira ES, Guimarães SB, Gadelha DD, Araujo Filho AD, Vasconcelos PRL. Alterações metabólicas, in vivo, induzidas por oclusão carotídea bilateral, no sangue e tecido ocular de ratos Wistar. Acta Cir Bras [serial online] 2003 Jul-Ago;18(4). Disponível em URL: http://www.scielo.br/acb.

RESUMO - Objetivo: Investigar as alterações metabólicas no olho e sangue arterial de ratos Wistar submetidos ao pinçamento bilateral das artérias carotídeas. Métodos: Trinta e seis ratos machos adultos foram submetidos a dissecção (grupo-1, sham, n=18) ou dissecção+pinçamento (grupo-2, experimento, $\mathrm{n}=18$ ) das artérias carotídeas comuns, sob anestesia geral. Após 30 minutos de isquemia (tempo 0) e durante a reperfusão (15-30 minutos) foram colhidas amostras de sangue arterial. Simultaneamente procedeu-se a enucleação do olho direito. Resultados: O aumento significante $(\mathrm{p}<0,05)$ das concentrações de piruvato tissular (tempo 0) no grupo-2 comparado ao controle sugere menor utilização do piruvato pelo tecido isquêmico. $\mathrm{O}$ aumento significante $(\mathrm{p}<0,05)$ do lactato tissular (grupo-2 versus grupo-1), no tempo 0, permanecendo elevado durante toda a fase de reperfusão, sugere uso continuado da via glicolítica anaeróbica, apesar da oferta de $\mathrm{O}_{2}$, nos tecidos lesados pela isquemia-reperfusão. Maior captação (grupo-1) pode ter sido a causa do aumento significante das concentrações de corpos cetônicos no olho, na reperfusão (15-30 minutos). Conclusão: A oclusão carotídea bilateral, durante 30 minutos, induz significativas alterações no metabolismo energético do tecido ocular, com manutenção da via glicolítica anaeróbica após restabelecimento do fluxo arterial.
\end{abstract}

DESCRITORES - Olho [metabolismo]. Isquemia. Lesão de reperfusão. Ratos.

\section{Introdução}

As doenças obstrutivas da artéria carotídea determinam uma isquemia ocular persistente com resultados devastadores para a visão, variando de cegueira permanente ou transiente, disfunção papilar até degeneração da retina ${ }^{1}$. Publicações recentes têm destacado o prognóstico e enfatizado o papel do oftalmologista no diagnóstico precoce destas afecções, assegurando aos pacientes portadores de obstrução da artéria carotídea um tratamento preventivo adequado, evitando suas complicações irreversíveis ${ }^{2}$. O processo de lesão e morte celular por isquemia tem despertado o espírito inquisidor dos pesquisadores. A isquemia resulta

1. Trabalho realizado no Laboratório de Cirurgia Experimental (LABCEX), Programa de Pós-Graduação Stricto Sensu em Cirurgia, Faculdade de Medicina, Departamento de Cirurgia da Universidade Federal do Ceará (UFC). Fortaleza - CE.

2. Professora Adjunta do Departamento de Morfologia. Mestranda em Cirurgia, do Programa de Pós-Graduação do Departamento de Cirurgia, UFC. Fortaleza - CE.

3. Mestre em Cirurgia. Professor Adjunto do Departamento de Cirurgia, Faculdade de Medicina (UFC). Fortaleza - CE.

4. Estudantes de Medicina (UFC).

5. Professor Doutor $(P h D)$, Coordenador do Curso de Pós-Graduação Stricto Sensu em Cirurgia da Faculdade de Medicina (UFC). Fortaleza - CE. 
do desequilíbrio entre a oferta de sangue arterial, rico em oxigênio, e as necessidades metabólicas dos tecidos. Isto produz alterações celulares diretamente relacionadas com a intensidade e duração da hipóxia tissular. Por outro lado, o retorno da perfusão sanguínea, após um prolongado período de isquemia, não é isento de efeitos deletérios, propiciando a ocorrência de danos adicionais ao tecido lesado pela isquemia. Durante a isquemia, a concentração intracelular de adenosina trifosfato (ATP) é reduzida, havendo então um aumento da concentração de adenosina monofosfato (AMP) que é metabolizada, posteriormente, em adenosina, iosina e hipoxantina. Após a reoxigenação dos tecidos, o oxigênio molecular reage com a hipoxantina e a xantina oxidase, produzindo superóxido, peróxido de hidrogênio e o radical hidroxila, metabólitos extremamente citotóxicos ${ }^{3}$.

Embora as diversas etapas do processo isquêmico ainda não estejam bem definidas, pela própria complexidade do processo, o fenômeno tem se mostrado uniforme e consistente, nos diversos modelos estudados, independentemente do tecido onde ocorre. Além disso, certos tecidos são mais suscetíveis à isquemia que outros. O sistema nervoso central é, entre todos os tecidos animais, o mais lábil ${ }^{4}$.

Objetivou-se neste trabalho estudar eventuais alterações induzidas pela isquemia/reperfusão, nas concentrações in vivo sangüíneas e nos tecidos oculares dos metabólitos (lactato, piruvato, glicose e corpos cetônicos) em ratos submetidos à obstrução carotídea bilateral seguida de reperfusão.

\section{Métodos}

A pesquisa foi realizada de acordo com as Normas Internacionais para a Pesquisa Biomédica em Animais (1990) e de acordo com a Lei Federal $n^{\circ} .6 .638$, de 08 de maio de $1979^{5}$. Utilizaram-se 36 ratos albinos (Rattus norvegicus albinus), da linhagem Wistar, machos, com peso oscilando entre 310 e 550 gramas, provenientes do Biotério Central da Universidade Federal do Ceará e mantidos no Laboratório de Cirurgia Experimental do Departamento de Cirurgia da Faculdade de Medicina (UFC) em gaiolas de polipropileno, recebendo água e alimento adequado ad libitum até 12 horas antes do início do experimento. Os ratos foram distribuídos eqüitativamente em dois grupos: Grupo 1 (controle) e Grupo 2 (experimental). Cada grupo foi subdividido em três subgrupos, $(n=6)$, de acordo com o período de coleta. Todos os procedimentos cirúrgicos foram realizados sob anestesia geral (cetamina $15 \mathrm{mg} / \mathrm{Kg}$ peso + xilasina $1 \mathrm{mg} / \mathrm{Kg}$ peso) administrada por via intraperitonial. Após identificação e isolamento das artérias carótidas comuns, aplicavam-se pinças vascular tipo buldogue, ocluíndo o fluxo sanguíneo, nos animais do grupo experimental (G-2). Nos animais do grupo controle (G-1), passava-se um fio de algodão 3-0 circundando as artérias, individualmente, sem ligaduras, removendo-o em seguida. Passados 30 minutos retiravam-se as pinças dos animais do G2, restabelecendose o fluxo arterial; ao término do período de isquemia (tempo 0) e 15 e 30 minutos mais tarde submetiam-se os diferentes subgrupos a uma laparotomia mediana para a coleta de 2,0 $\mathrm{ml}$ de sangue arterial (aorta abdominal). Simultaneamente, o olho direito era removido por enucleação. A coleta de amostras de sangue e tecido ocular dos animais do grupo controle obedeceu à mesma sistemática, iniciada 30 minutos após a dissecção das artérias (Operação Simulada ou Sham).

Os metabólitos no sangue da aorta e no tecido ocular direito foram determinados por métodos de ensaio enzimático ${ }^{6,7,8}$. Foram aferidas neste estudo as concentrações in vivo dos precursores da gliconeogênese (piruvato e lactato), dos corpos cetônicos (acetoacetato e 3-hidroxibutirato) e a da D-glicose. As concentrações dos metabólitos foram calculadas em micromol/g tecido fresco ou micromol/ $\mathrm{ml}$ de sangue.

Os resultados foram expressos como Média \pm \pm E.P.M. (Erro Padrão da Média). O teste não paramétrico de Mann-Whitney foi utilizado para a análise estatística, fixando-se o valor de significância em 5\% ( $<<0,05)$.

\section{Resultados}

Houve queda significante na glicemia 30 minutos após o início da reperfusão em relação aos tempos 0 e 15 minutos, nos animais submetidos à isquemia (Figura 1).

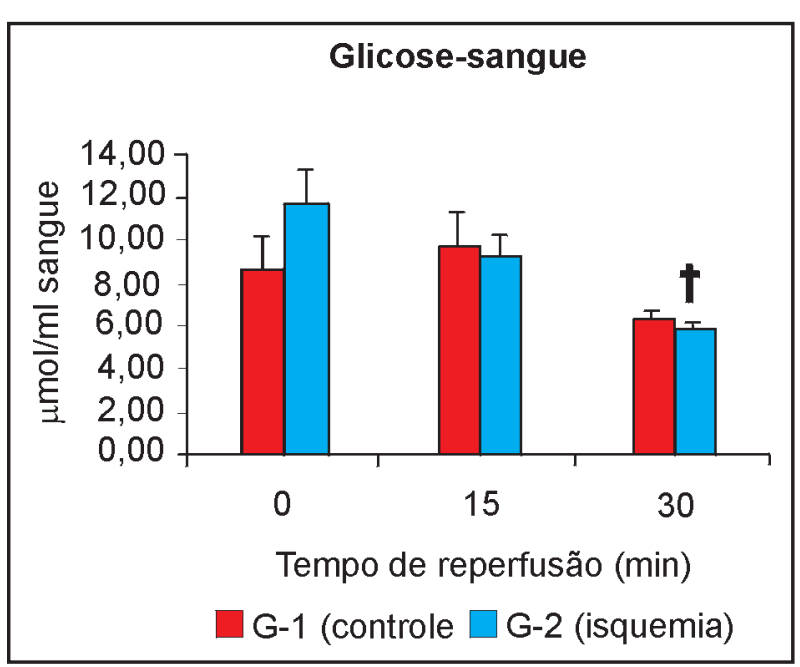

$\dagger \mathrm{p}<0,01$ quando comparado aos tempos 0 e 15 min, no mesmo grupo

FIGURA 1 - Concentração de glicose no sangue dos ratos. 
Não foram observadas diferenças nas concentrações de glicose no olho dos ratos em nenhum dos tempos estudados. Por outro lado, houve aumento significante da concentração de piruvato no sangue dos animais isquêmicos, quando comparados aos respectivos controles, ao término do período de isquemia (tempo 0). Houve decréscimo nas concentrações de piruvato nos tempos 15 e 30 minutos no mesmo grupo em relação ao tempo $\mathrm{O}$ (Figura 2). Observou-se ainda redução significante da concentração de piruvato no olho dos animais do grupo 2, 30 minutos após o início da reperfusão, em relação aos tempos 0 e 15 minutos, no mesmo grupo (Figura 3).

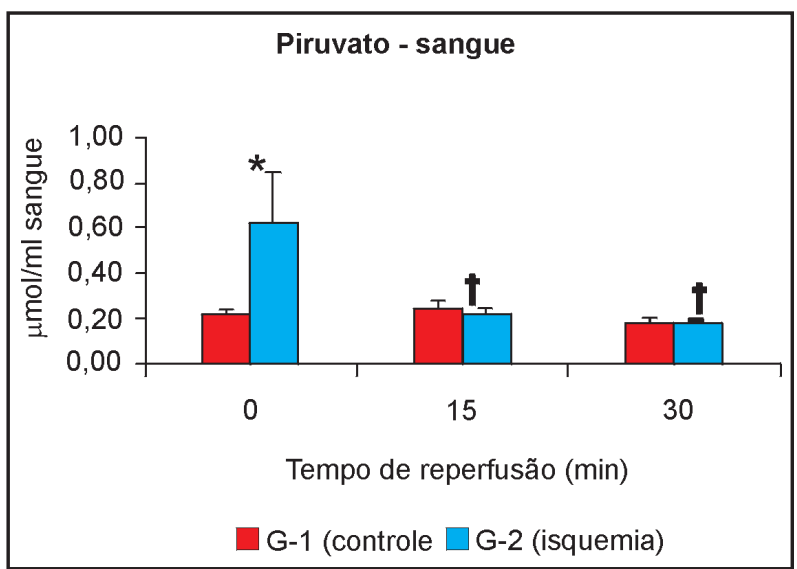

* p $<0,05$ quando comparado ao respectivo controle (tempo 0)

$\dagger \mathrm{p}<0,05$ quando comparado ao tempo 0 no mesmo grupo

FIGURA 2 - Concentrações de piruvato no sangue ( $\mu \mathrm{mol} / \mathrm{ml})$.

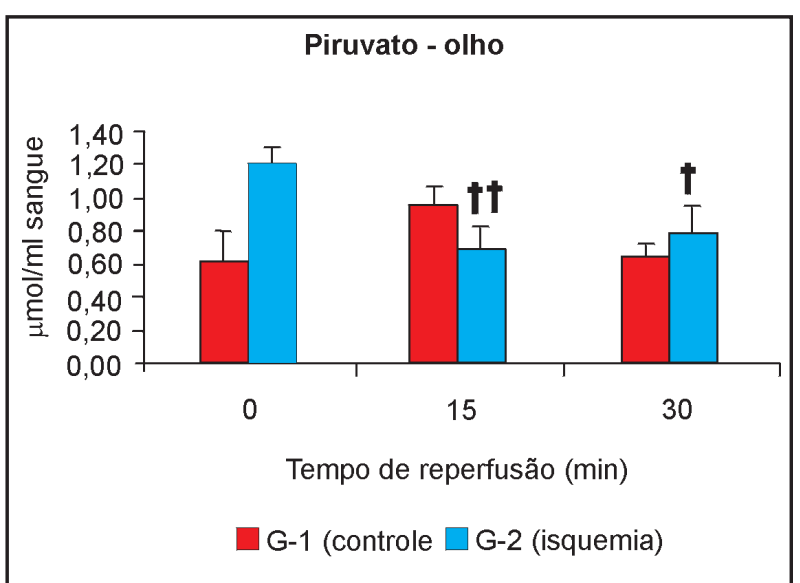

$\dagger \mathrm{p}<0,05$ quando comparado ao tempo 15 min no mesmo grupo $\dagger \mathrm{p}<0,01$ quando comparado ao tempo 0 min no mesmo grupo

FIGURA 3 - Concentrações de piruvato no olho $(\mu \mathrm{mol} / \mathrm{g}$ tec. fresco).

Houve aumento significante na lactacemia 15 minutos após o início da reperfusão nos ratos do grupo controle, em relação ao tempo 0 (Figura 4)

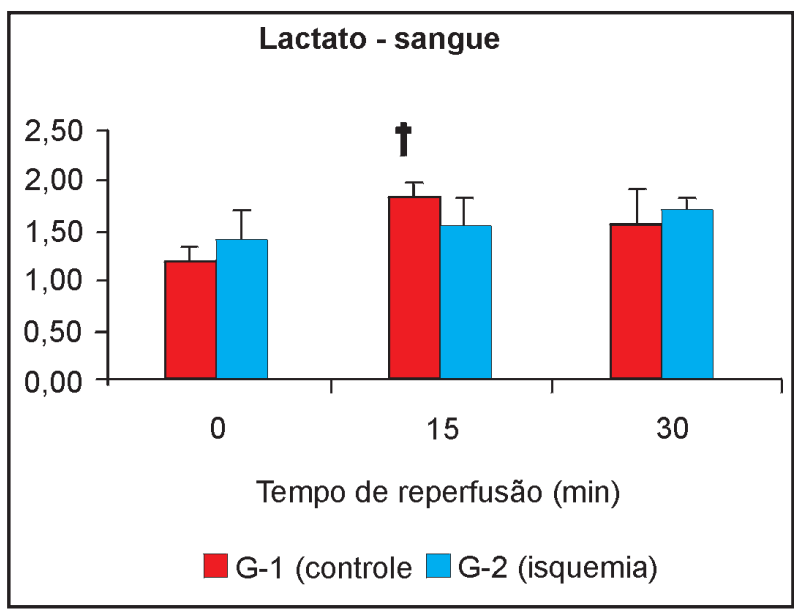

$\dagger \mathrm{p}<0,05$ quando comparado ao tempo 0 no mesmo grupo

FIGURA 4 - Concentrações de lactato no sangue $(\mu \mathrm{mol} / \mathrm{ml})$.

Verificou-se um aumento significante da concentração de lactato no olho dos ratos do grupo controle 15 minutos após o início da reperfusão. Houve também incremento significante das concentrações de lactato em tecido ocular isquêmico (tempo 0 ) em relação ao tecido do olho de animais do grupo controle (Figura 5).

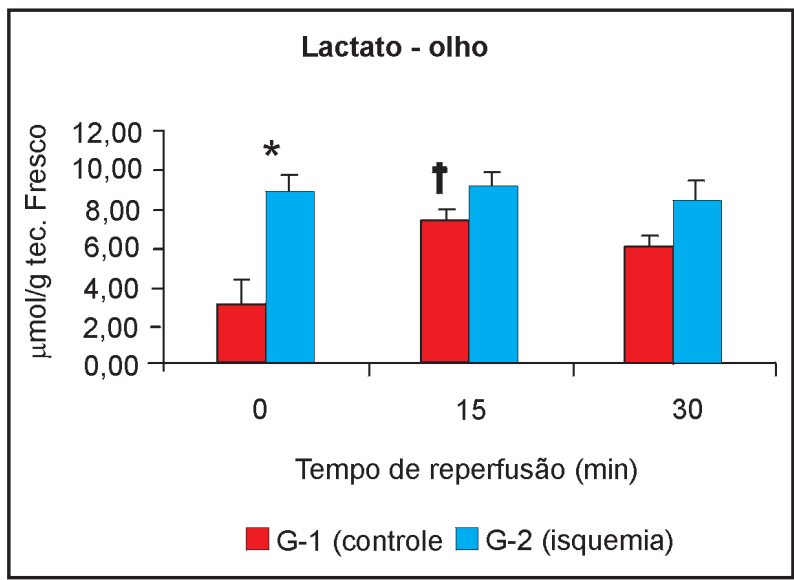

$* \mathrm{p}<0,01$ quando comparado ao respectivo controle (tempo 0 ) $\dagger \mathrm{p}<0,05$ quando comparado ao tempo 0 no mesmo grupo

FIGURA 5 - Concentrações de lactato no olho ( $\mu \mathrm{mol} / \mathrm{g}$ tec. fresco).

Não houve alteração na concentração de acetoacetato e 3-hidroxibutirato no sangue dos ratos, em nenhum dos grupos estudados. Houve aumento significante da concentração de acetoacetato no olho dos animais do grupo controle 30 minutos após o início da reperfusão, em relação ao tempo 15 minutos (Figura 6). Não houve alteração na concentração de 3-hidroxibutirato no sangue dos ratos, em nenhum dos grupos estudados. 


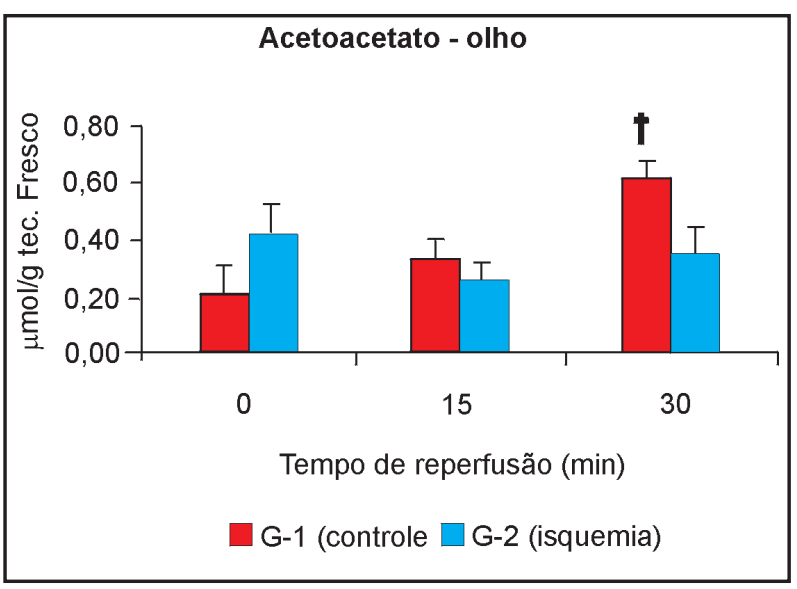

$\dagger \mathrm{p}<0,05$ quando comparado aos tempos 0 e 15 min no mesmo grupo

FIGURA 6 - Concentrações de lactato no olho ( $\mu \mathrm{mol} / \mathrm{g}$ tec. fresco).

Não houve alteração na concentração de corpos cetônicos no sangue dos ratos, em nenhum dos grupos estudados. Houve aumento significante da concentração de corpos cetônicos 30 minutos após o início da reperfusão em relação ao tempo 0 , no olho dos ratos do grupo controle. Houve ainda aumento significante da concentração de corpos cetônicos 15 minutos após o início da reperfusão em relação ao tempo 0 , no mesmo grupo (Figura 7).

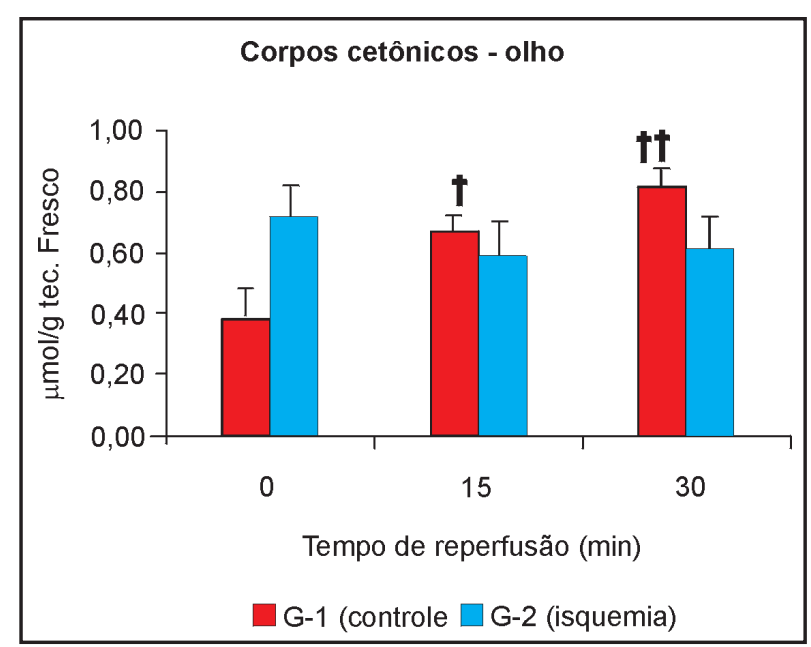

$\dagger \mathrm{p}<0,05$ quando comparado ao tempo 0 no mesmo grupo $\dagger \mathrm{p}<0,01$ quando comparado ao tempo 0 no mesmo grupo

FIGURA 7 - Concentrações de corpos cetônicos no olho ( $\mu$ mol/ $\mathrm{g}$ tec. fresco).

\section{Discussão}

O modelo de isquemia utilizado no presente estudo, com bloqueio bilateral das artérias carotídeas por 30 minutos, produziu alterações metabólicas significantes. Trabalhos recentes utilizaram tempos de isquemia variando de 30 até 120 minutos, realizando diferentes procedimentos para a obtenção da isquemia. Rosembaum et al. (2001), estudando os efeitos da isquemia retiniana transiente, decorrente de obstrução da artéria retiniana por aumento da pressão intraocular, encontraram alterações com redução no número de células retinianas após 30 minutos de isquemia. As alterações retinianas se tornavam mais pronunciadas após uma hora de isquemia. ${ }^{9}$

O trauma determina alterações graves no metabolismo, com consumo acelerado das reservas de glicose oriundas do glicogênio hepático e muscular. Como os tecidos estritamente dependentes da glicose (retina, hemácias e medula renal) mantêm a demanda crescente desse nutriente, ao mesmo tempo em que o aporte de glicose exógena encontra-se reduzido, torna-se necessário a produção desse metabólito a partir de outros substratos (gliconeogênese). O lactato pode ser utilizado nessa via metabólica, no fígado, sendo convertido a piruvato pela enzima lactato desidrogenase e entrando na cadeia gliconeogênica (ciclo de Cori). $\mathrm{Na}$ fase catabólica de resposta ao trauma, além do lactato, estão aumentadas as concentrações hepáticas e sanguíneas de alanina e piruvato, este último produto da desaminação da alanina, elevada pela quebra de proteínas.

Lafuente et al. (2002) submeteram ratos SpragueDawley adultos à isquemia retinal transiente por ligaduras dos vasos oftálmicos. Os períodos de isquemia variaram de 30 a 120 minutos. Todos os períodos de isquemia produziram perda das células ganglionares da retina. Ratos submetidos à isquemia de 30 minutos apresentaram destruição e morte de $46 \%$ das células ganglionares retinianas. Estudos metabólicos não foram, no entanto, realizados. ${ }^{10}$

Diante do exposto, seria presumível que o modelo utilizado no presente estudo, com isquemia do olho decorrente do pinçamento bilateral das artérias carotídeas, poderia produzir resultados ainda mais expressivos.

A redução significante da glicemia $(\mathrm{p}<0,05)$ observada nos animais submetidos à oclusão carotídea bilateral 30 minutos após o restabelecimento da perfusão sanguínea em relação aos tempos 0 e 15 minutos, pode ser decorrente de uma maior captação de glicose pelos tecidos periféricos para glicólise, ou menor produção de glicose pelo fígado.

No presente estudo, a redução significante da concentração sanguínea de piruvato 15 e 30 minutos pós-perfusão em relação ao tempo 0 , no grupo de ratos submetidos à isquemia, sugere maior utilização desse 
metabólito para a produção de energia, pelos tecidos periféricos. O mesmo comportamento ocorreu nas concentrações deste metabólito nos tecidos oculares sujeitos à isquemia nos tempos 15 e 30 minutos de reperfusão.

O aumento significante da lactacemia aos 15 minutos de reperfusão em relação ao tempo 0 , nos animais do grupo controle pode ser explicado pelo efeito trauma. Entretanto, o mesmo fenômeno não correu nos ratos submetidos à isquemia. $\mathrm{O}$ aumento da concentração de lactato no tecido ocular dos animais submetidos à isquemia ratifica a eficácia do modelo utilizado no presente estudo. A elevação da concentração de lactato no tecido isquêmico foi $280 \%$ superior àquela aferida no grupo controle (tempo 0). $\mathrm{O}$ aumento significante $(\mathrm{p}<0,05)$ do lactato tissular (grupo-2 versus grupo-1), no tempo 0 , permanecendo elevado durante toda a fase de reperfusão, sugere uso continuado da via glicolítica anaeróbica, apesar da oferta de $\mathrm{O} 2$, nos tecidos lesados pela isquemia-reperfusão.

Os corpos cetônicos são importantes fontes alternativas de energia formadas por uma via bioquímica específica no fígado, a partia do acetil-CoA. É evidente a importância dos dois principais corpos cetônicos, o acetoacetato e o 3-hidroxibutirato, no papel de combustíveis metabólicos. Em humanos, o jejum costuma induzir elevação dos níveis desses substratos no sangue, como forma de adaptação metabólica para a preservação da vida. ${ }^{11}$ Não foram verificadas alterações nas concentrações sanguíneas de acetoacetato. Entretanto, houve aumento das concentrações de acetoacetato nos tecidos oculares 30 minutos no período pós-operatório, em animais do grupo controle, sugerindo aumento de captação, já que a cetonemia era semelhante em todos os tempos estudados (grupo 1). O mesmo ocorreu com relação às concentrações de corpos cetônicos no tempo 30 minutos no mesmo grupo de animais (grupo controle), também sugerindo maior captação destes metabólitos por tecido ocular não submetido à isquemia/ reperfusão. Tal elevação não ocorreu em tecidos oculares submetidos à isquemia/reperfusão talvez em decorrência de lesão tissular residual 30 minutos após reperfusão, com menor capacidade de captação dos corpos cetônicos.

\section{Conclusão}

A oclusão carotídea bilateral, durante 30 minutos, induz significativas alterações no metabolismo energético do tecido ocular, com manutenção da via glicolítica anaeróbica após restabelecimento do fluxo sanguíneo arterial.

\section{Referências}

1. Stevens WD, Fortin T, Pappas BA. Retinal and optic nerve degeneration after chronic carotid ligation: time course and role of light exposure. Stroke 2002; 33 (4): 1107-12.

2. Biousse V. Carotid disease and the eye. Curr Opin Ophtalmol 1997; 8 (6): 16-26.

3. McCord JM. Oxygen-derived free radicals in postischemic tissue injury. N Eng J Med 1985; 312 (3): 159-63.

4. Ames AIII, Nesbitt FB. In vitro retina as an experimental model of the central nervous system. J Neurochem 1991; 37:867-77.

5. Brasil. Lei Federal n. 6.638, de 8 de maio de 1979. Estabelece normas para a prática didático-científica da vivissecção de animais e determina outras providências. In Brasil. Coleção de leis de 1979: atos legislativos do poder executivo. Leis de abril a junho. Brasília, Departamento de Imprensa Nacional, 1979. p 33-4.

6. Hohorst apud Vasconcelos PRL. Hepatic metabolism during sepsis [Tese - Doutorado]. University Oxford; 1987.

7. Williamson DH, Mellanby J, Krebs HA. Enzymic determinations of the D-(-) â-hydroxybutyric acid and acetoacetic acid in blood. Biochem J, 1962; 82: 90-6.

8. Slein apud Vasconcelos PRL. Hepatic metabolism during sepsis [Tese - Doutorado]. University Oxford; 1987.

9. Rosenbaum DM, Rosembaum PS, Singh M, Gupta G, Gupta H, Li B, Roth S. Functional and morphological comparison of two methods to produce transient retinal ischemia in the rat. J Neuroophtalmol 2001; 21 (1): 62-8.

10. Lafuente MP, Villegas-Peres MP, Selles-Navarro I, MayorTorroglosa S, Mirales de Imperial J, Vidal-Sanz M. Retinal ganglion cell death after acute retinal ischemia is an ongoing process whose severity and duration depends on the duration of the insult. Neuroscience 2002; 109 (1): 157-68.

11. Figueiredo PHD, Vasconcelos, PRL. Adaptative hiperketonemia upon intra venous infusion of lipid emulsions in fasted septic patient. Int Care Med 1998; 24 (supl. 1): S48. 
Oliveira ES, Guimarães SB, Gadelha DD, Araujo Filho AD, Vasconcelos PRL. Metabolic alterations, in vivo, induced by bilateral carotid artery occlusion upon the blood and eye tissue of Wistar rats. Acta Cir Bras [serial online] 2003 Jul-Aug;18(4). Available from URL: http://www.scielo.br/acb.

ABSTRACT - Purpose: To investigate the metabolic alterations in the eye and arterial blood of Wistar rats submitted to bilateral common carotid artery clamping. Methods: thirty-six males adult rats were submitted the dissection (group-1, sham, $\mathrm{n}=18$ ) or dissection+clamping (group-2, experimental, $\mathrm{n}=18$ ) of common carotid arteries, under general anesthesia. After 30 minutes of ischemia (time 0) and during reperfusion (15-30 minutes) arterial blood samples were collected. Simultaneously right eye enucleation was carried out. Results: The significant decrease of tissue piruvate concentrations $15 \mathrm{~min}(0,693 \pm 0,132$ versus $1,195 \pm 0,118 \mathrm{mmol} / \mathrm{g}, \mathrm{p}<0,01)$ and $30 \mathrm{~min}$ $(0,764 \pm 0,182$ versus $1,195 \pm 0,118 \mathrm{mmol} / \mathrm{g}, \mathrm{p}<0,05)$ of onset of reperfusion (time 0$)$ in group-2 rats suggests increased use of piruvate for energy production in Krebs cycle. The significant increase of tissue lactate (group-2 versus group-1), in time $0(8,843 \pm 0,902$ versus $3,134 \pm 1,182$ $\mathrm{mmol} / \mathrm{g}, \mathrm{p}<0,01)$ remaining elevated during the whole reperfusion phase, suggests continuous use of the anaerobic glicolytical pathway, in spite of the offer of $\mathrm{O} 2$, in ischemia-reperfusion injured tissues. Increased uptake (group-1) might have been the cause for the significant increase of ketone bodies concentrations in the eye, during reperfusion (15-30 minutes). Conclusion: Bilateral carotid artery occlusion, for 30 minutes, induces significant alterations in the energetic metabolism of eye tissue, with maintenance of the anaerobic glicolytical pathway after re-establishment of the arterial flow.

KEY WORDS - Eye [metabolism]. Ischemia. Reperfusion lesion. Rats.

Conflito de interesse: nenhum

Fonte de financiamento: nenhuma

Correspondência:

Paulo Roberto Leitão de Vasconcelos

Departamento de Cirurgia

Rua Prof. Costa Mendes, 1608/3 andar

60430-140 Fortaleza - CE

Tel: (85)288-8063 - Fax: (85)288-8064

mcirur@npd.ufc.br

Data do recebimento: 15/03/2003

Data da revisão: 23/03/2003

Data da aprovação: 04/04/2003 